\title{
Exercise flow-volume loops in prepubescent aerobically trained children
}

\author{
Cédric Nourry, ${ }^{1}$ Fabien Deruelle, ${ }^{2}$ Claudine Fabre, ${ }^{2}$ Georges Baquet, ${ }^{2}$ Frédéric Bart, ${ }^{3}$ \\ Jean-Marie Grosbois, ${ }^{3}$ Serge Berthoin, ${ }^{2}$ and Patrick Mucci ${ }^{1,2}$ \\ ${ }^{1}$ Laboratoire d'Analyse Multidisciplinaire des Pratiques Sportives, Unité de Formation et de Recherche des Sciences \\ et Techniques des Activités Physiques et Sportives de Liévin, Université d'Artois, Chemin du Marquage, Liévin; ${ }^{2}$ Laboratoires \\ d'Etudes de la Motricité Humaine EA 3608, Faculté des Sciences du Sport et de l'Education Physique, Université de Lille 2, \\ Ronchin; ${ }^{3}$ Service de pneumologie, Hôpital Germon-Gauthier de Béthune-Beuvry, Beuvry, France
}

Submitted 21 March 2005; accepted in final form 4 July 2005

\begin{abstract}
Nourry, Cédric, Fabien Deruelle, Claudine Fabre, Georges Baquet, Frédéric Bart, Jean-Marie Grosbois, Serge Berthoin, and Patrick Mucci. Exercise flow-volume loops in prepubescent aerobically trained children. J Appl Physiol 99: 1912-1921, 2005. First published July 7, 2005; doi:10.1152/japplphysiol.00323.2005.—We studied mechanical ventilatory constraints in 13 aerobically trained (Tr) and 11 untrained (UT) prepubescent children by plotting the exercise flow-volume (F-V) loops within the maximal F-V loop (MFVL) measured at rest. The MFVL allowed to determine forced vital capacity (FVC) and maximal expiratory flows. Expiratory and inspiratory reserve volumes relative to FVC (ERV/FVC and IRV/ FVC, respectively) were measured during a progressive exercise test until exhaustion. Breathing reserve (BR) and expiratory flow limitation (expFL), expressed in percentage of tidal volume (VT) and defined as the part of the tidal breath meeting the boundary of the MFVL, were measured. Higher FVC and maximal expiratory flows were found in $\operatorname{Tr}$ than UT $(P<0.05)$ at rest. Our results have shown that during exercise, excepting one subject, all $\mathrm{Tr}$ regulated their VT within FVC similarly during exercise, by breathing at low lung volume at the beginning of exercise followed breathing at high lung volume at strenuous exercise. In UT, ERV/FVC and IRV/FVC were regulated during exercise in many ways. The proportion of children who presented an expFL was nearly the same in both groups $(\sim 70 \%$ with a range of 14 to $65 \%$ of $\mathrm{VT}$ ), and no significant difference was found during exercise concerning expFL. However, higher ventilation ( $\mathrm{VE}$ ), ERV/FVC, and dyspnea associated with lower BR, IRV/FVC, and $\mathrm{Sa}_{2}$ were reported at peak power in $\operatorname{Tr}$ than UT $(P<0.05)$. These results suggest that, because of their higher $\mathrm{VE}$ level, trained children presented higher ventilatory constraints than untrained. These may influence negatively the $\mathrm{Sa}_{\mathrm{O}_{2}}$ level and dyspnea during strenuous exercise.
\end{abstract}

mechanical ventilatory constraints; expiratory limitation; dynamical hyperinflation; training; healthy children

PULMONARY FUNCTION IS GENERALLY not limited during exercise in healthy young adults. However, highly fit men (22) or women (27), who reach high ventilation levels, may present mechanical ventilatory constraints. Indeed, athletes are able to greatly increase their oxygen uptake $\left(\dot{\mathrm{V}}_{2}\right)$ with training, but the adaptability of the lung capacity is much less than that of the systemic vasculature or cardiac and skeletal muscle (11).

The consequence of this difference in adaptation processes with training is a conflict between the ventilatory demand and capacity $(11,12)$. This results in occurrence of ventilatory constraints and sometimes in pulmonary gas-exchange impair-

Address for reprint requests and other correspondence: C. Nourry, UFR des STAPS de Liévin, Laboratoire d'Analyse Multidisciplinaire des Pratiques et Sportives, Chemin du Marquage, 62800 Liévin, France (e-mail: nourryce @wanadoo.fr). ment during exercise, which may be partly implicated in a decrease in arterial oxygen saturation $\left(\mathrm{Sa}_{\mathrm{O}_{2}}\right)(22)$.

The ventilatory constraints may be evidenced by the measured of the breathing reserve (BR). To our knowledge, there are only a few studies reporting values of BR in children (30, 31). A low BR was found at peak exercise in the children who developed gas-exchange impairment during exercise (30), suggesting a high level of ventilatory constraints in some children. In addition, the measure of BR does not bring precise information about the origin of the ventilatory constraints (23). An accurate method to analyze ventilatory constraints during exercise consists in plotting exercise flow-volume (F-V) loops within a maximal flow-volume loop (MFVL) measured at rest (23). This method allows to evidence the existence and the amplitude of both expiratory flow limitation (expFL) and dynamical hyperinflation, which reflect the level of the ventilatory constraints $(5,23)$. First, expFL is determined by a partial encroachment of the tidal breath on the MFVL. This was evidenced in trained subjects, more especially during strenuous exercise $(4,20,21,27)$. Second, in adults, ventilatory limitation generally influences the regulation of the tidal volume (VT) within the total lung capacity during exercise $(22,23$, 28). In fit adults, when the minute ventilation ( $\left.\mathrm{V}_{\mathrm{E}}\right)$ and the VT increase with exercise, tidal breaths encroach the limit defined by the MFVL and $\mathrm{V}_{\text {T }}$ is shifted toward the high lung volume. This phenomenon is called dynamical hyperinflation $(21,22,27)$.

Consequently, much of the mechanical constraint on $\dot{V}_{E}$ appears to be imposed by airway diameter limiting the flow rate $(20,22,28)$, as suggested by studies that reported significant increase in $\dot{V E}$ when subjects breathed low-density $\mathrm{He}_{-} \mathrm{O}_{2}$ mixtures to expand the maximum F-V envelope and to avoid expFL $(4,28)$. Moreover, some studies have shown that women are particularly affected by ventilatory constraints (27) or gas-exchange impairment during exercise $(17,18)$, because of smaller lungs and airways for the same body size than men $(18,27,29)$. Children also have smaller airways relative to lung size than adults (29) and ventilate excessively to the metabolic demands of exercise $(2,8,15,32)$. Hence, we hypothesized that trained children who are able to reach a high level of $\dot{\mathrm{V}}_{2}$ (26) could be particularly predisposed to ventilatory constraints because of high $\dot{V}$ E. In trained children, the higher $\dot{V}_{E}$ may impose breathing at higher lung volume and consequently at a flatter part of the compliance curve, one requiring more work of breathing (23).

\footnotetext{
The costs of publication of this article were defrayed in part by the payment of page charges. The article must therefore be hereby marked "advertisement" in accordance with 18 U.S.C. Section 1734 solely to indicate this fact.
} 
Therefore, the aim of this study was to assess expiratory flow limitation and to determine how Vт is regulated within FVC during exercise in trained and untrained prepubescent children, by plotting exercise F-V loops within MFVL.

\section{MATERIALS AND METHODS}

Subjects. Twenty-four prepubescent children participated in this study. A physical activity questionnaire validated for French children (10) was distributed to the parents to assess the physical activity of each child. Thirteen sportive children $(10.8 \pm 1.1 \mathrm{yr}$ old; 4 girls and 9 boys) composed the first group (Tr). They were enrolled in aerobic sports (athletics, swimming, tennis) and had an extracurricular sporting activity $>5 \mathrm{~h} / \mathrm{wk}(7.9 \pm 2.4 \mathrm{~h})$. Eleven untrained children $(10.5 \pm$ $1.0 \mathrm{yr}$ old; 4 girls and 7 boys) formed the second group (UT). The untrained children had low extracurricular sporting activity $(0.7 \pm$ $1.0 \mathrm{~h}$ ) or none. A complete clinical checkup was carried out by a physician to search for contraindications to exercise in all subjects. All subjects were free of cardiac or respiratory diseases including exercise-induced asthma. The subjects' body mass, height, body mass index, and fat mass percentage, estimated with the skinfold method, were measured (13). The same physician verified that each subject was in the first stage of maturation according to Tanner's method (35). The experimental protocol was approved by the local Ethics Committee. All subjects and their parents received a verbal description of the experiment and completed a written, informed consent form.

Lung function tests. Children, equipped with a nose clip, performed two lung function tests to measure MFVL (Ergocard, Medi-soft, Dinant, Belgium). The first lung function test was completed before the graded exercise test, and the second was carried out 10 min after the end of exercise. All lung function tests were carried out by the same technician and followed the guidelines of the American Thoracic Society (1). For each lung function test, three MFVL were taken to calculate forced vital capacity (FVC), forced expiratory volume in $1 \mathrm{~s}\left(\mathrm{FEV}_{1}\right)$, peak expiratory flow (PEF), and maximal expiratory flow at $75 \%\left(\mathrm{MEF}_{75 \%}\right), 50 \%\left(\mathrm{MEF}_{50 \%}\right)$, and $25 \%\left(\mathrm{MEF}_{25 \%}\right)$ of FVC. Only the MFVL with the largest combination of $\mathrm{FEV}_{1}$ and FVC was retained.

Graded exhaustive exercise test. The subjects performed a graded exercise test to exhaustion on a magnetic-braked cycle ergometer (800 S, Ergoline, Bitz, Germany). The graded exercise test began with a resting period of 5 min and continued with a 3-min warm-up period at $10 \mathrm{~W}$. Exercise intensity was then increased by steps of $10 \mathrm{~W}$ each minute until exhaustion so that exercise lasted between 8 and $10 \mathrm{~min}$. Subjects had to remain upright while maintaining a pedaling rate of 60 $\mathrm{rpm}$ and were verbally encouraged until exhaustion.

Gas exchanges and cardiorespiratory measurements. Throughout exercise, a breath-by-breath gas analyzer (Ergocard, Medi-soft), cal-

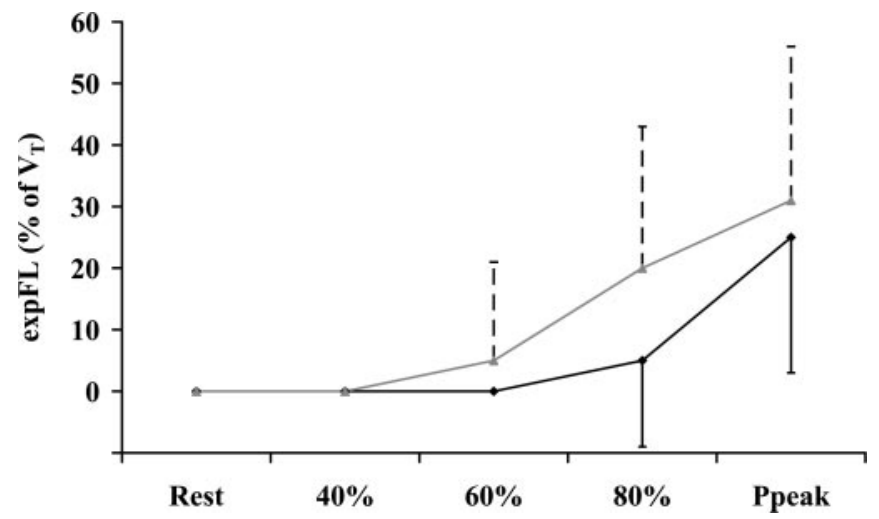

Fig. 1. Expiratory flow limitation (expFL) during exercise in trained ( $\square$ ) and untrained children $(\mathbf{\Delta})$. Ppeak, peak power.
Table 1. Subject characteristics

\begin{tabular}{lcc}
\hline \hline & $\operatorname{Tr}(n=13)$ & $\mathrm{UT}(n=11)$ \\
\hline Age, yr & $10.8 \pm 1.1$ & $10.5 \pm 1.0$ \\
Body mass, kg & $36.4 \pm 7.2$ & $40.9 \pm 14.1$ \\
Height, cm & $144.9 \pm 8.1$ & $142.5 \pm 9.9$ \\
Fat mass, \% & $9.1 \pm 6.3^{*}$ & $19.9 \pm 9.4$ \\
BMI, kg/m ${ }^{2}$ & $17.2 \pm 1.6$ & $19.8 \pm 4.9$ \\
{$[\mathrm{Hb}], \mathrm{g} / \mathrm{dl}$} & $13.5 \pm 1.1$ & $13.0 \pm 1.3$ \\
\hline
\end{tabular}

Values are means $\pm \mathrm{SD}$. Tr, trained children; UT, untrained children; BMI, body mass index; $[\mathrm{Hb}]$, hemoglobin concentration. Significant difference between $\mathrm{Tr}$ and $\mathrm{UT}: * P<0.05$.

ibrated before each test with references gases, was used to measure $\dot{\mathrm{V}}_{2}$, carbon dioxide output $\left(\dot{\mathrm{V}}_{\mathrm{CO}_{2}}\right)$, end-tidal carbon dioxide pressure, end-tidal oxygen pressure, $\dot{\mathrm{V}}$, $\mathrm{V}_{\mathrm{T}}$, breathing frequency $(f)$, mean inspiratory flow $\left(\mathrm{V}_{\mathrm{T}} / \mathrm{T}_{\mathrm{I}}\right)$, and duty cycle ( $\mathrm{T}_{\mathrm{I}} / \mathrm{Tt}$ tot). All values were averaged over 30-s periods. BR was estimated from maximal voluntary ventilation (MVV) and $\dot{V}$ e reached at maximal exercise, according to the following equation (23):

$$
\mathrm{BR}(\%)=\left[\left(\mathrm{MVV}-\dot{\mathrm{V}}_{\mathrm{E}}\right) / \mathrm{MVV}\right] \times 100
$$

where MVV was determined from the following formula (33):

$$
\mathrm{MVV}=27.7 \times \mathrm{FEV}_{1}+8.8 \times \text { predicted } \mathrm{FEV}_{1}
$$

Predicted values of $\mathrm{FEV}_{1}$ were determined here according the prediction equation elaborated by Knudson et al. (25) for children.

Expired volume was measured at mouth with a pneumotachograph, which was calibrated with a 3-liter calibration syringe. Subjects breathed through a pediatric mouthpiece maintained with a helmet.

Ventilatory response to exercise $\left(\Delta \dot{\mathrm{V}} / \Delta \dot{\mathrm{V}}_{\mathrm{CO}_{2}}\right)$ was individually determined from the slope of the relationship, measured for each subject by linear regression analysis, between $\dot{\mathrm{V}}$ and $\dot{\mathrm{V}}_{\mathrm{CO}_{2}}$ recorded between the onset of exercise and occurrence of the respiratory compensation point (30).

During the graded exercise, the heart rate was recorded by means of a 12-lead electrocardiogram (Ergocard, Medi-soft). At each minute during exercise, a dyspnea rating scale (7) was shown to the subjects, who were asked to point out a score with a finger. Values of $\mathrm{Sa}_{\mathrm{O}_{2}}$ were obtained by a noninvasive method, i.e., a pulse ear oximeter $(3800 \mathrm{~S}$, Datex Ohmeda, Madison, WI). Before exercise, the ear was warmed first with a vasodilator ointment, and the probe was kept in place on the ear by means of adhesive tape to avoid potential problems in signal recording. Moreover, the pulse oximeter evaluated waveform signal quality pulse by pulse, indicating when problems associated with blood flow to the ear might influence $\mathrm{Sa}_{\mathrm{O}_{2}}$. Before exercise, $\mathrm{Sa}_{\mathrm{O}_{2}}$ was recorded and averaged over $3 \mathrm{~min}$, whereas during exercise, $\mathrm{Sa}_{\mathrm{O}_{2}}$ was recorded during the last $10 \mathrm{~s}$ of each minute only when the accuracy of the signal was optimal. The pulse oximeter was automatically calibrated before each experiment.

Blood lactate measurement. At rest, before exercise, and immediately after the end of the graded exercise test, fingertip blood samples of $10 \mu \mathrm{l}$ were collected to measure hemoglobin and blood lactate concentrations by spectrophotometric analysis method (Miniphotometer LP 20, Dr. Lange, Düsseldorf, Germany).

Determination of flow limitation and breathing strategy. To assess variations in breathing strategy during exercise and to determine how close children came to an expFL, tidal F-V loops were recorded during the graded exercise test using a bidirectional flow-sensing pneumotachograph coupled to the gas analyzer (Ergocard, Medi-soft). The tidal F-V loops were plotted during exercise within MFVL determined before exercise (Fig. 1). MFVL and exercise tidal F-V loops were recorded with the same apparatus. A mean of 10-20 tidal breaths immediately preceding a maximal inspiratory capacity (IC) maneuver was recorded over the last $30 \mathrm{~s}$ of each stage of exercise. Maximal IC maneuvers were carried out after collection of the tidal 
Table 2. Lung function test data

\begin{tabular}{lll}
\hline \hline & $\operatorname{Tr}(n=13)$ & $\mathrm{UT}(n=11)$ \\
\hline FVC, liters & $2.78 \pm 0.62^{*}$ & $2.32 \pm 0.57$ \\
$\mathrm{FEV}_{1}$, liter & $2.35 \pm 0.48^{*}$ & $1.86 \pm 0.45$ \\
$\mathrm{FEV}_{1} / \mathrm{FVC}, \%$ & $85.2 \pm 7.5$ & $84.5 \pm 12.0$ \\
$\mathrm{PEF}, 1 / \mathrm{s}$ & $4.95 \pm 1.09^{*}$ & $3.63 \pm 1.23$ \\
$\mathrm{MEF}_{75 \%}, 1 / \mathrm{s}$ & $4.51 \pm 0.87^{*}$ & $3.42 \pm 1.17$ \\
$\mathrm{MEF}_{50 \%}, 1 / \mathrm{s}$ & $3.17 \pm 0.48^{*}$ & $2.50 \pm 0.86$ \\
$\mathrm{MEF}_{25 \%}, 1 / \mathrm{s}$ & $1.53 \pm 0.29 \dagger$ & $1.38 \pm 0.46$ \\
\hline
\end{tabular}

Values are means \pm SD. FVC, forced vital capacity; $\mathrm{FEV}_{1}$, forced expiratory volume in $1 \mathrm{~s} ; \mathrm{PEF}$, peak expiratory flow; $\mathrm{MEF}_{75 \%, 50 \%}$, and $25 \%$, maximal expiratory flow at 75,50 , and $25 \%$ of FVC, respectively. Significant difference between $\operatorname{Tr}$ and $\mathrm{UT}: * P<0.05, \dagger P<0.01$.

breaths at each workload to correct for drift in the F-V sensing devices (23). The tidal breaths were averaged by a computer averaging program to provide a representative tidal F-V loop with appropriate placement in MFVL for each workload. The expFL was defined as the part of the F-V loops that met the boundary of the expiratory portion of the MFVL determined before exercise and was expressed in percentage of the exercise $\mathrm{V}_{\mathrm{T}}$. The regulation of $\mathrm{V}_{\mathrm{T}}$ within FVC during exercise was reflected by the changes in end-expiratory and end-inspiratory lung volume (EELV and EILV, respectively). EELV and EILV were estimated from, respectively, expiratory and inspiratory reserve volume relative to FVC (ERV/FVC and IRV/FVC, respectively) $(6,23)$.

Statistical analysis. All values are given as means \pm SD. The statistical analysis was performed using statistical software (SigmaStat 2.03, SPSS Science, Chicago, IL). Normality was checked before each treatment. A two-way repeated-measures ANOVA was used to compare the values of each parameter between both groups and to compare the change from rest in each group (group $\times$ intensity). A linear regression test was used to search for correlations between the different parameters. The level of significance was set at $P<0.05$.

\section{RESULTS}

All the subjects were at the first stage of maturation. Table 1 presents the subject characteristics. The mean value of weekly physical activity was higher in $\operatorname{Tr}$ than in UT $(P<0.01)$. No significant difference was reported between Tr and UT concerning all the anthropometrical data except for body fat mass percentage, which was lower in $\operatorname{Tr}(P<0.05)$ (Table 1$)$.

Spirometrical data. Table 2 shows the lung function test data measured at rest before exercise. Mean MFVL is globally higher in Tr than UT with higher values reported for FVC $(P<$ $0.05), \operatorname{FEV}_{1}(P<0.05), \operatorname{PEF}(P<0.05), \mathrm{MEF}_{75 \%}(P<0.05)$, $\mathrm{MEF}_{50 \%}(P<0.05)$, and $\mathrm{MEF}_{25 \%}(P<0.01)$ in Tr. Postexercise values of spirometrical data were not significantly different from preexercise values in each group.

Comparison of resting and exercise metabolic parameters. Table 3 presents the metabolic parameters. Throughout exercise, Tr presented higher values than UT of $\dot{\mathrm{V}}_{2}$ power, $\dot{\mathrm{V}}_{\mathrm{CO}_{2}}$ from 40 , 60 , and $80 \%$, respectively, up to peak exercise (Ppeak) $(P<$ 0.05). No difference was found during submaximal exercise concerning $\Delta \dot{\mathrm{V}} \mathrm{E} / \Delta \dot{\mathrm{V}}_{\mathrm{CO}_{2}}$ between $\mathrm{Tr}$ and UT $(28.3 \pm 2.8$ vs. $29.3 \pm 1.6$, respectively). At Ppeak, higher values of $\dot{\mathrm{V}} \mathrm{E} / \dot{\mathrm{V}}_{2}$ were found in Tr than UT $(P<0.05)$, whereas no significant difference was reported between both groups concerning $\dot{\mathrm{V}} / \dot{\mathrm{V}}_{\mathrm{CO}_{2}}$. Higher end-tidal oxygen pressure and lower end-tidal carbon dioxide pressure were found at Ppeak in Tr than UT $(P<0.05)$. Lower $\mathrm{Sa}_{\mathrm{O}_{2}}$ was reported from $80 \%$ up to Ppeak in Tr than UT $(P<$ $0.05)$. At Ppeak, values of blood lactate concentration were $9.6 \pm$ 3.7 and $7.0 \pm 2.2$ in $\mathrm{Tr}$ and UT, respectively.

Ventilatory and timing parameters at rest and throughout exercise. Table 4 shows the ventilatory and timing parameters. At rest, no difference was observed concerning $\dot{V}_{\mathrm{E}}, f, \mathrm{~V}_{\mathrm{T}}$, TI/Ttot, VT/FVC, dyspnea, and BR between both groups.

Table 3. Resting and exercise metabolic parameters

\begin{tabular}{|c|c|c|c|c|c|}
\hline & Rest & $40 \%$ of Ppeak & $60 \%$ of Ppeak & $80 \%$ of Ppeak & Ppeak \\
\hline $\operatorname{Tr}$ & & $54.1 \pm 10.9$ & $80.8 \pm 16.7 *$ & $107.7 \pm 22.2 *$ & $134.6 \pm 27.8^{*}$ \\
\hline UT & & $43.3 \pm 10.2$ & $65.9 \pm 15.3$ & $86.6 \pm 20.3$ & $107.7 \pm 25.1$ \\
\hline $\operatorname{Tr}$ & $9.0 \pm 2.4$ & $24.7 \pm 4.4 *$ & $32.5 \pm 3.6 \dagger$ & $42.1 \pm 5.1 \dagger$ & $50.4 \pm 4.0 \dagger$ \\
\hline UT & $9.7 \pm 3.5$ & $20.6 \pm 9.1$ & $26.0 \pm 4.1$ & $31.0 \pm 5.2$ & $36.1 \pm 4.9$ \\
\hline \multicolumn{6}{|c|}{$\dot{\mathrm{V}} \mathrm{CO}_{2} 1 / \mathrm{min}$} \\
\hline \multicolumn{6}{|c|}{ PET $_{\mathrm{O}_{2}}$, Torr } \\
\hline $\operatorname{Tr}$ & $106.0 \pm 3.6$ & $103.7 \pm 3.6$ & $106.9 \pm 3.1$ & $110.5 \pm 3.8$ & $118.5 \pm 3.6^{*}$ \\
\hline UT & $104.4 \pm 7.2$ & $104.3 \pm 3.4$ & $106.7 \pm 2.2$ & $110.4 \pm 3.6$ & $113.7 \pm 3.2$ \\
\hline \multicolumn{6}{|c|}{$\mathrm{PETCO}_{2}$, Torr } \\
\hline $\operatorname{Tr}$ & $37.9 \pm 3.8$ & $37.9 \pm 3.8$ & $37.4 \pm 3.1$ & $36.2 \pm 2.8$ & $30.4 \pm 3.3 *$ \\
\hline UT & $35.2 \pm 2.7$ & $38.9 \pm 2.9$ & $38.4 \pm 1.7$ & $37.1 \pm 2.6$ & $35.6 \pm 2.4$ \\
\hline \multicolumn{6}{|c|}{$\dot{\mathrm{V}} \mathrm{E} / \mathrm{V} \mathrm{O}_{2}$} \\
\hline \multicolumn{6}{|c|}{$\mathrm{Sa}_{2}, \%$} \\
\hline $\operatorname{Tr}$ & $98.0 \pm 0.4$ & $96.9 \pm 0.8$ & $96.5 \pm 0.9$ & $95.9 \pm 0.8 *$ & $94.5 \pm 1.7^{*}$ \\
\hline UT & $97.8 \pm 0.7$ & $97.1 \pm 0.6$ & $96.6 \pm 0.5$ & $96.7 \pm 0.5$ & $96.3 \pm 1.1$ \\
\hline
\end{tabular}

Values are means \pm SD. Ppeak, peak power. Exercise intensity is expressed in percentage of Ppeak. $\mathrm{VO}_{2}$, oxygen consumption; $\mathrm{V}_{\mathrm{CO}_{2}}$, carbon dioxide output; $\mathrm{PET}_{2}$, end-tidal partial oxygen pressure; $\mathrm{PET}_{\mathrm{CO}_{2}}$, end-tidal partial carbon dioxide pressure; $\mathrm{SaO}_{2}$, arterial oxygen saturation; $\mathrm{V} E$, minute ventilation. Significant difference between $\operatorname{Tr}$ and UT: $* P<0.05, \uparrow P<0.01$. 
Table 4. Ventilatory and timing variables during exercise

\begin{tabular}{|c|c|c|c|c|c|}
\hline $\operatorname{Tr}$ & $12.3 \pm 3.69$ & $26.8 \pm 4.28$ & $37.5 \pm 6.7$ & $52.7 \pm 10.7 *$ & $79.7 \pm 17.9 *$ \\
\hline $\operatorname{Tr}$ & $17.3 \pm 4.6$ & $31.6 \pm 8.2$ & $35.6 \pm 6.8$ & $43.2 \pm 8.7$ & $60.9 \pm 9.8^{*}$ \\
\hline UT & $20.6 \pm 5.0$ & $33.6 \pm 3.5$ & $39.2 \pm 6.8$ & $44.2 \pm 8.3$ & $50.6 \pm 6.1$ \\
\hline \multicolumn{6}{|c|}{ VT, liters } \\
\hline \multicolumn{6}{|c|}{ TI/Ttot } \\
\hline $\operatorname{Tr}$ & $0.46 \pm 0.20$ & $0.46 \pm 0.04$ & $0.46 \pm 0.02$ & $0.47 \pm 0.02$ & $0.50 \pm 0.02$ \\
\hline UT & $0.48 \pm 0.16$ & $0.44 \pm 0.04$ & $0.45 \pm 0.04$ & $0.48 \pm 0.03$ & $0.46 \pm 0.09$ \\
\hline \multicolumn{6}{|c|}{$\mathrm{VT} / \mathrm{FVC}, \%$} \\
\hline $\operatorname{Tr}$ & $30.0 \pm 8.5$ & $34.7 \pm 6.6$ & $41.0 \pm 7.2$ & $46.8 \pm 7.1$ & $49.7 \pm 6.4$ \\
\hline UT & $26.2 \pm 7.0$ & $34.6 \pm 4.4$ & $39.5 \pm 6.1$ & $47.1 \pm 7.9$ & $52.5 \pm 7.4$ \\
\hline \multicolumn{6}{|c|}{ VT/Ti, 1/s } \\
\hline \multicolumn{6}{|c|}{ Dyspnea } \\
\hline $\operatorname{Tr}$ & $0 \pm 0$ & $1.7 \pm 1.1$ & $2.9 \pm 1.5$ & $5.0 \pm 1.7$ & $7.5 \pm 1.6^{*}$ \\
\hline UT & $0 \pm 0$ & $1.6 \pm 1.6$ & $3.2 \pm 2.6$ & $4.2 \pm 2.6$ & $5.9 \pm 3.0$ \\
\hline
\end{tabular}

Values are means $\pm \mathrm{SD}$. Exercise intensity is expressed in percentage of Ppeak. $f$, Breathing frequency; VT, tidal volume; Ti/Ttot, duty cycle; VT/Ti, mean inspiratory flow; VT/FVC, VT relative to forced vital capacity; BR, breathing reserve. Significant difference between $\operatorname{Tr}$ and $\mathrm{UT}$ : $* P<0.05, \dagger P<0.01$.

During exercise, higher $\dot{V} \mathrm{E}$ were reported from $80 \%$ up to Ppeak $(P<0.05)$ associated with higher $f$ at Ppeak in Tr than UT $(P<0.05)$, but no significant difference was found concerning VT (Table 4). No significant difference was reported between both groups concerning $\mathrm{T}_{\mathrm{I}} / \mathrm{Ttot}$ and $\mathrm{VT}_{\mathrm{T}} / \mathrm{FVC}$ throughout exercise. Higher values of $\mathrm{VT}_{\mathrm{T}}$ TI were reported at Ppeak in Tr than UT $(P<0.05)$. At Ppeak only, Tr presented higher dyspnea $(P<0.05)$ and lower BR $(P<0.01)$ than UT.

Table 5 presents the F-V loop parameters measured during exercise. Higher values of ERV were found at rest and at Ppeak in Tr than UT. No significant difference was found between the two groups concerning IRV, IC, and difference between exercise and resting values of IC $(\Delta \mathrm{IC})$ throughout exercise.

The values of IRV/FVC, VT/FVC, and ERV/FVC measured at 50 and $75 \mathrm{~W}$ are reported in the Table 6. No significant difference was found concerning the parameters at these two specific exercise intensities.
Exercise $F$-V loops. Figures 1 and $2, A$ and $B$, illustrate changes in expFL, ERV/FVC, and IRV/FVC, respectively, during exercise. No significant difference was reported in expFL between $\mathrm{Tr}$ and UT from rest up to Ppeak (Fig. 1). Nine trained and eight untrained children presented an expFL during exercise. During exercise, mean ERV/FVC did not change in UT, whereas in Tr, it decreased from rest at 40 and $60 \%(P<0.01)$ (Fig. $2 A)$ before coming back near resting mean value at $80 \%$ and Ppeak. Concerning IRV/FVC, a decrease from rest was reported from $60 \%$ up to Ppeak in UT and at Ppeak only in $\operatorname{Tr}(P<0.01)$ (Fig. 2B). At rest, higher ERV/FVC $(P<0.05)$ and lower IRV/FVC were found in Tr than UT $(P<0.01)$ (Fig. 2). Higher ERV/FVC and lower IRV/FVC were reported at Peak in Tr than UT $(P<0.05)$.

Individual responses. The degree of flow limitation and the regulation of VT within ERV and IRV consecutive to the increase in $\dot{V} \mathrm{E}$ were quite variable among subjects. Consequently, individual responses of tidal breathing within MFVL

Table 5. Flow-volume loop parameters during exercise

\begin{tabular}{|c|c|c|c|c|c|}
\hline & Rest & $40 \%$ of Ppeak & $60 \%$ of Ppeak & $80 \%$ of Ppeak & Ppeak \\
\hline $\operatorname{Tr}$ & $0.92 \pm 0.38$ & $1.03 \pm 0.27$ & $0.91 \pm 0.24$ & $0.70 \pm 0.19$ & $0.56 \pm 0.09$ \\
\hline UT & $1.12 \pm 0.31$ & $0.97 \pm 0.34$ & $0.86 \pm 0.30$ & $0.73 \pm 0.35$ & $0.61 \pm 0.27$ \\
\hline $\operatorname{Tr}$ & $0.99 \pm 0.73^{*}$ & $0.73 \pm 0.32$ & $0.68 \pm 0.33$ & $0.71 \pm 0.28$ & $0.84 \pm 0.38 *$ \\
\hline UT & $0.52 \pm 0.24$ & $0.51 \pm 0.29$ & $0.56 \pm 0.27$ & $0.52 \pm 0.26$ & $0.53 \pm 0.28$ \\
\hline \multicolumn{6}{|c|}{ IC, liters } \\
\hline \multicolumn{6}{|c|}{$\Delta \mathrm{IC}$, liters } \\
\hline $\operatorname{Tr}$ & & $0.25 \pm 0.42$ & $0.30 \pm 0.42$ & $0.25 \pm 0.47$ & $0.12 \pm 0.43$ \\
\hline UT & & $0.03 \pm 0.25$ & $0.04 \pm 0.22$ & $0.01 \pm 0.22$ & $0.01 \pm 0.24$ \\
\hline
\end{tabular}

Values are means \pm SD. IRV and ERV, inspiratory and expiratory reserve volumes, respectively; IC, inspiratory capacity; $\Delta \mathrm{IC}$, values of IC at rest are subtracted to values of IC measured during exercise. Significant difference between $\operatorname{Tr}$ and UT: $* P<0.05$. 
Table 6. Flow-volume loop parameters at the same absolute exercise intensity in $\operatorname{Tr}$ and $U T$

\begin{tabular}{lll}
\hline \hline & \multicolumn{1}{c}{$50 \mathrm{~W}$} & $75 \mathrm{~W}$ \\
\hline VE $1 / \mathrm{min}$ & & \\
$\mathrm{Tr}$ & $26.8 \pm 3.1$ & $34.4 \pm 3.9$ \\
$\mathrm{UT}$ & $27.6 \pm 3.6$ & $36.9 \pm 4.1$ \\
$\mathrm{IRV} / \mathrm{FVC}, \%$ & $39.9 \pm 6.1$ & $35.8 \pm 9.9$ \\
$\mathrm{Tr}$ & $40.4 \pm 11.6$ & $36.7 \pm 11.4$ \\
$\mathrm{UT}$ & $33.9 \pm 5.1$ & $39.0 \pm 5.8$ \\
$\mathrm{VT} / \mathrm{FVC}, \%$ & $36.3 \pm 6.1$ & $43.3 \pm 7.4$ \\
$\mathrm{Tr}$ & & \\
UT & $23.6 \pm 7.0$ & $26.9 \pm 7.0$ \\
ERV/FVC, \% & $22.5 \pm 11.1$ & $22.3 \pm 8.0$ \\
Tr &
\end{tabular}

Values are means \pm SD. IRV/FVC and ERV/FVC, inspiratory and expiratory reserve volume relative to forced vital capacity, respectively.

are presented in Figs. 3 and 4 for each trained and untrained children, respectively. In Tr group, nine children presented an expFL at peak exercise within a range of 14 to $60 \%$ of VT. In three children (subjects 2,3, and 12) of the nine presenting a ventilatory limitation, expFL began at $80 \%$ of Ppeak. Except for one subject (subject 7), all the trained children regulated their VT within ERV and IRV similarly during exercise. Indeed, 12 trained children presented a decrease in EELV (reflected by an increase in ERV/FVC) followed by an increase in this parameter (Fig. 3) except for subject 7. In this subject, an increase in $\mathrm{V}_{\mathrm{T}}$ consecutive to the increase in $\mathrm{V}_{\mathrm{E}}$ was shared out between IRV and ERV up to peak exercise, and EELV decreased continuously during the entire exercise test (Fig. 3).

In UT group, eight subjects presented an expFL ranging between 15 and $65 \%$. Among these flow-limiting children, expFL began at $80 \%$ in five children (subjects $15,17,21,22$, and 23) and from $40 \%$ only in one child (24), with a mean value ranging between 15 and $47 \%$ of VT. During exercise, untrained children regulated their $\mathrm{V}_{\mathrm{T}}$ within FVC in many ways. Indeed, six subjects (subjects 16, 18, 19,23, and 24) of 11 regulated their $\mathrm{V}_{\text {T }}$ similarly than the majority of the trained children during exercise as presented above. In the five remaining children of this group, the regulation on $\mathrm{V}_{\text {T }}$ during exercise was quite variable, as illustrated by Fig. 4. In subject 14, a significant increase in EELV was observed between rest and $80 \%$ of Ppeak followed by a decrease at peak exercise. In subject 15, EELV decreased until $40 \%$ of Ppeak, increased between 40 and $60 \%$, decreased between 60 and $80 \%$, and then increased up to peak exercise. Subject 17 presented a decrease in EELV between rest and $40 \%$ of Ppeak followed by an increase between 40 and $60 \%$ and a decrease from $80 \%$ up to peak exercise again. Subject 20 presented an increase in EELV between rest and $40 \%$ of Ppeak and then decrease from $40 \%$ up to peak exercise. In subject 21, EELV decreased until $40 \%$ Ppeak, increased from 40 to $80 \%$, and then decreased until peak exercise.

Relationships between respiratory parameters. Figures 5-7 show the significant relationships when the two groups were gathered together. Significant relationships were reported between peak $\dot{\mathrm{V}}_{2}$ and PEF, $\mathrm{MEF}_{75 \%}$ and $\mathrm{MEF}_{50 \%}(n=24 ; P<$ $0.01 ; r=0.44, r=0.46$, and $r=0.51$, respectively). Resting values of ERV/FVC were significantly related to PEF and $\mathrm{MEF}_{75 \%}(n=24 ; P<0.05 ; r=0.49$ and $r=0.43$, respectively) (Fig. 5). Significant relationships were reported between peak exercise values of ERV/FVC and $\mathrm{FEV}_{1}, \mathrm{PEF}$, and $\mathrm{MEF}_{75 \%}(n=24 ; P<0.05 ; r=0.51, r=0.46$, and $r=$ 0.50 , respectively) (Fig. 6). Peak exercise values of ERV/FVC were significantly related to peak exercise values of $\dot{V}_{\mathrm{E}}(n=$ 24; $P<0.05 ; r=0.46$ ) (Fig. 7), and a significant relationship was found between peak exercise values of IRV/FVC and VT/Ti $(n=24 ; P<0.01 ; r=-0.58)$.

\section{DISCUSSION}

The aim of this work was to compare the ventilatory constraints between trained and untrained prepubescent children. First, we found that Tr presented lower BR and higher dyspnea at Ppeak. Secondly, we reported higher ERV/FVC and lower IRV/FVC at Ppeak in Tr than UT, suggesting a breath at higher lung volume in $\mathrm{Tr}$.

Maximal resting F-V loops. In this study, we reported significant larger MFVL (greater FVC and maximal expiratory flows) in Tr than UT, which suggests that training could improve pulmonary function in children. In the literature, the beneficial "fitness effect" on MFVL in children is not clearly established. Studies have suggested that long-term swim training could improve lung volume or even maximal expiratory flows $(3,9,37,38)$, whereas fitness effect on MFVL function in land-based activities is still a matter of debate $(14,16,34)$. In our study, 8 trained children of 13 were enrolled in competitive aquatic activities (swimming and swimming with flippers), which could explain partly the difference between both groups. Moreover, we reported that peak $\dot{\mathrm{V}}_{2}$ was slightly but significantly related to $\mathrm{PEF}, \mathrm{MEF}_{75 \%}$, and $\mathrm{MEF}_{50 \%}$, which suggests that the trained children have the greater expiratory flows. Similar results were previously reported in a fit aging population by Johnson et al. (21), which supports our findings.

Ventilatory flow limitation. Our results show lower values of $\mathrm{BR}$ in $\mathrm{Tr}$ compared with UT, suggesting that ventilatory constraints are more important in the first group. To our
Fig. 2. Flow-volume loop parameters during exercise in trained (๘) and untrained children $(\mathbf{\Lambda})$ : expiratory reserve volume relative to forced vital capacity (ERV/FVC) $(A)$, and inspiratory reserve volume relative to forced vital capacity (IRV/FVC) (B). The changes in end-expiratory and end-inspiratory lung volume (EELV and EILV, respectively) during exercise are evidenced by changes in ERV/ FVC and IRV/FVC, respectively. Significant difference between groups: $* P<0.05$, $* * P<0.01$. Significant difference from rest: $\ddagger P<0.01$.
A

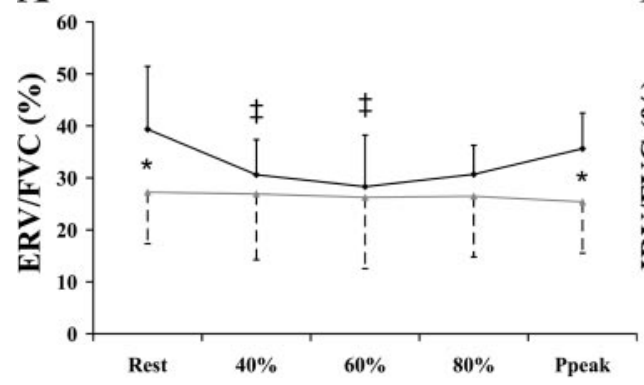

B

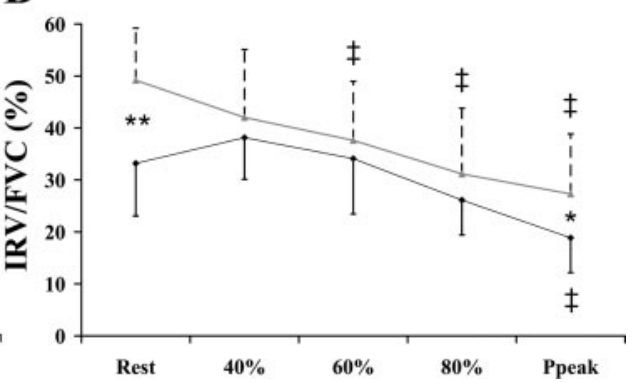



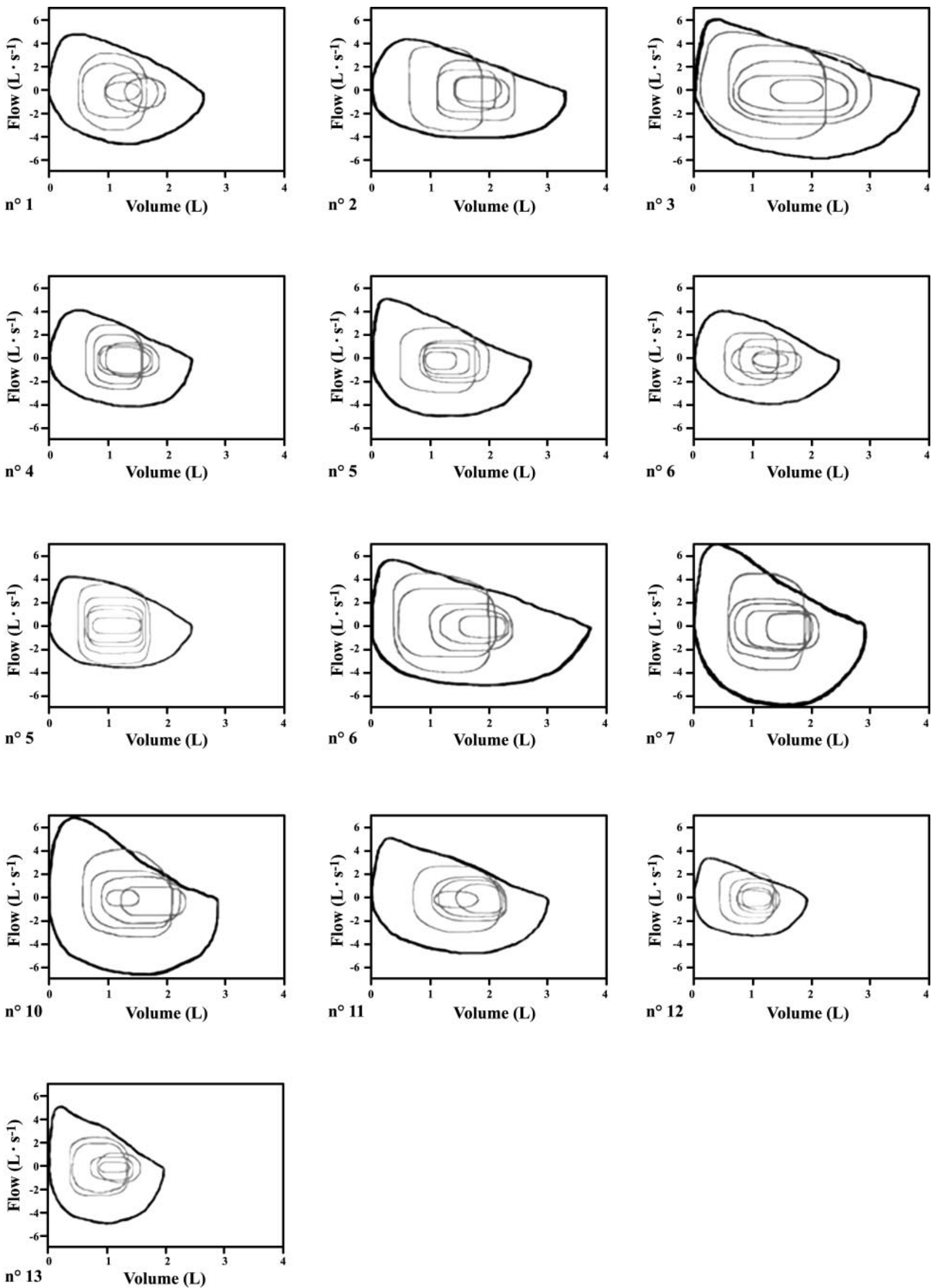

Fig. 3. Individual exercise flow-volume loops plotted within the maximal flow-volume loop measured at rest in trained children. 

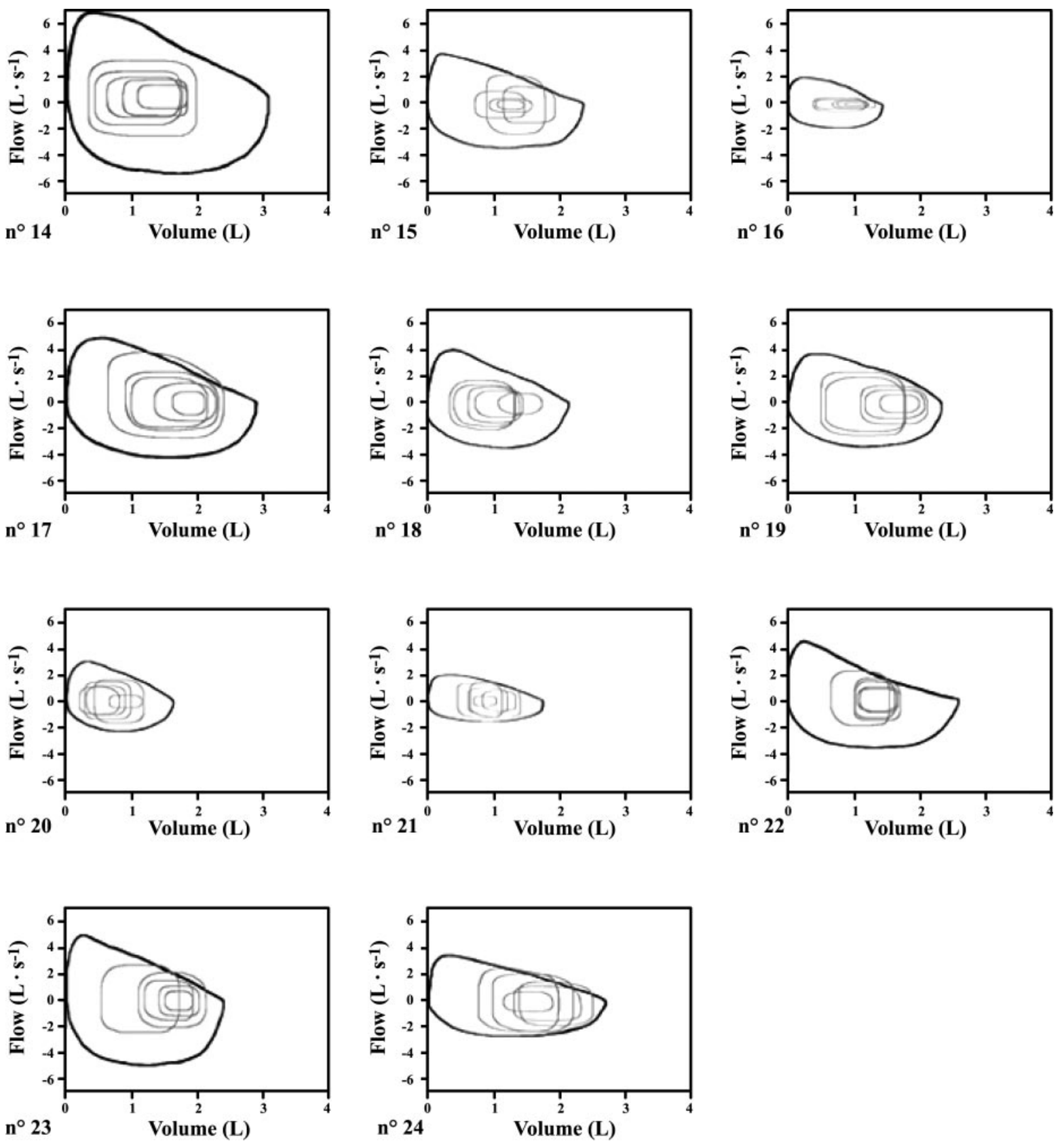

Fig. 4. Individual exercise flow-volume loops plotted within the maximal flow-volume loop measured at rest in untrained children.

knowledge, there is no other comparison of BR between $\mathrm{Tr}$ and UT in children in the literature. In addition, 17 prepubescent children of 24 presented an expFL ranging between 14 and $65 \%$ of $\mathrm{V}$ т at peak exercise. But one other interesting finding in this study was that the proportion of children who presented an expFL was nearly the same in $\operatorname{Tr}(9$ of 13) and UT ( 8 of 11) children. At first sight, this result is in contrast with our hypothesis of higher ventilatory constraints in $\mathrm{Tr}$ than UT children. However, as explain below, the peak exercise expFL is associated with higher EELV and EILV in Tr. In adults, expFL during exercise is generally observed in endurance-trained athletes, in old fit subjects, or in patients suffering from pulmonary disease, reflecting ventilatory constraints because of conflict between ventilatory demand and pulmonary system capacity $(5,20-23,27)$. However, McClaran et al. (27) also reported expFL during exercise in low fit women, which supports our results showing that expFL may exist in both healthy untrained and trained children. The prevalence of expFL in children whatever their training status, compared with adults, could be explained by the facts that 1 ) children are known to ventilate excessively to the metabolic demands of exercise $(8,15)$ and 2) they have small airways relative to lung size (29). In consequence, the relatively high ventilation levels in children might more easily reach the mechanical limit of respiratory system. Thus the expFL may be explained by a larger MFVL combined with a greater ventilatory drive in $\mathrm{Tr}$, whereas UT simply have a smaller MFVL. 

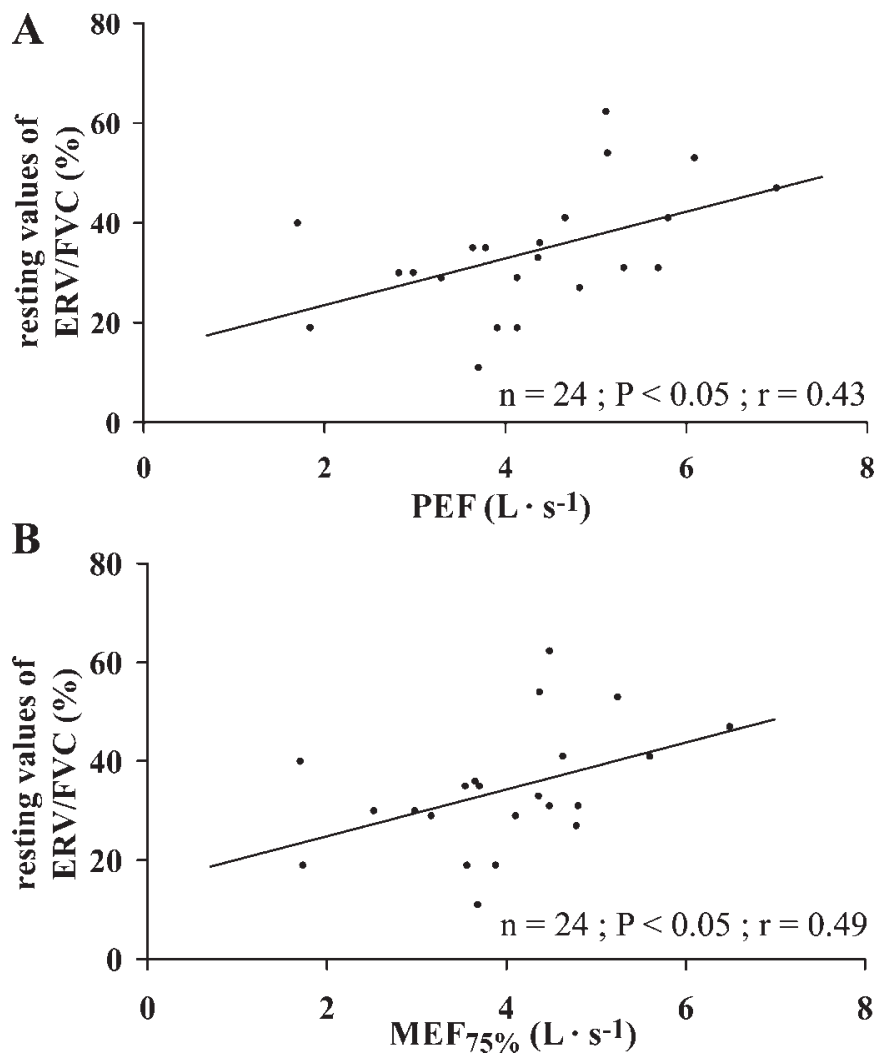

Fig. 5. A: significant relationship between resting values of ERV/FVC and maximal expiratory flow at $75 \%$ of forced vital capacity $\left(\mathrm{MEF}_{75 \%}\right)$. B: significant relationship between resting values of ERV/FVC and peak expiratory flow (PEF).

Changes in dynamical lung volumes and breathing pattern during exercise. To attenuate the heterogeneity in lung volume in the children population, we investigated dynamical lung volume (EELV and EILV) by determining ERV and IRV relative to FVC (see MATERIALS AND METHODS).

At rest, our results show that EELV and EILV are yet higher in $\operatorname{Tr}$ than UT. Johnson et al. (20) have previously found a difference in expiratory flows rates and EELV between the most fit and the least fit subjects in an aging population. In our study, the resting values of ERV/FVC were significantly related to PEF and $\mathrm{MEF}_{75 \%}$. These results suggest that maximal expiratory flows may be implicated in the difference in resting values of ERV/FVC between both groups. Courteix et al. (9) have shown that airway resistance and maximal expiratory flows do not evolve in the same way in controls and girl swimmers after $1 \mathrm{yr}$ of training. Hence, they suggested that swim training may interact with maturation process of the lung. In consequence, we can suppose that fitness effects on airway resistance may contribute to modify the place of $\mathrm{V}_{\mathrm{T}}$ within $\mathrm{FVC}$ at rest in trained children, maybe because of a change in the balance between airway resistance and lung compliance.

During exercise, the similar $\Delta \dot{\mathrm{V}}_{\mathrm{E}} / \Delta \dot{\mathrm{V}}_{\mathrm{CO}_{2}}$ in both groups shows that $\mathrm{Tr}$ and UT seem to regulate arterial carbon dioxide pressure at the same carbon dioxide set point, whereas they present difference in exercise ventilation (8). With the exception of one subject (subject 7), all the trained children presented the same breathing pattern during exercise. At first, $\mathrm{Tr}$ decreased EELV (reflected by a decrease in ERV/FVC) until
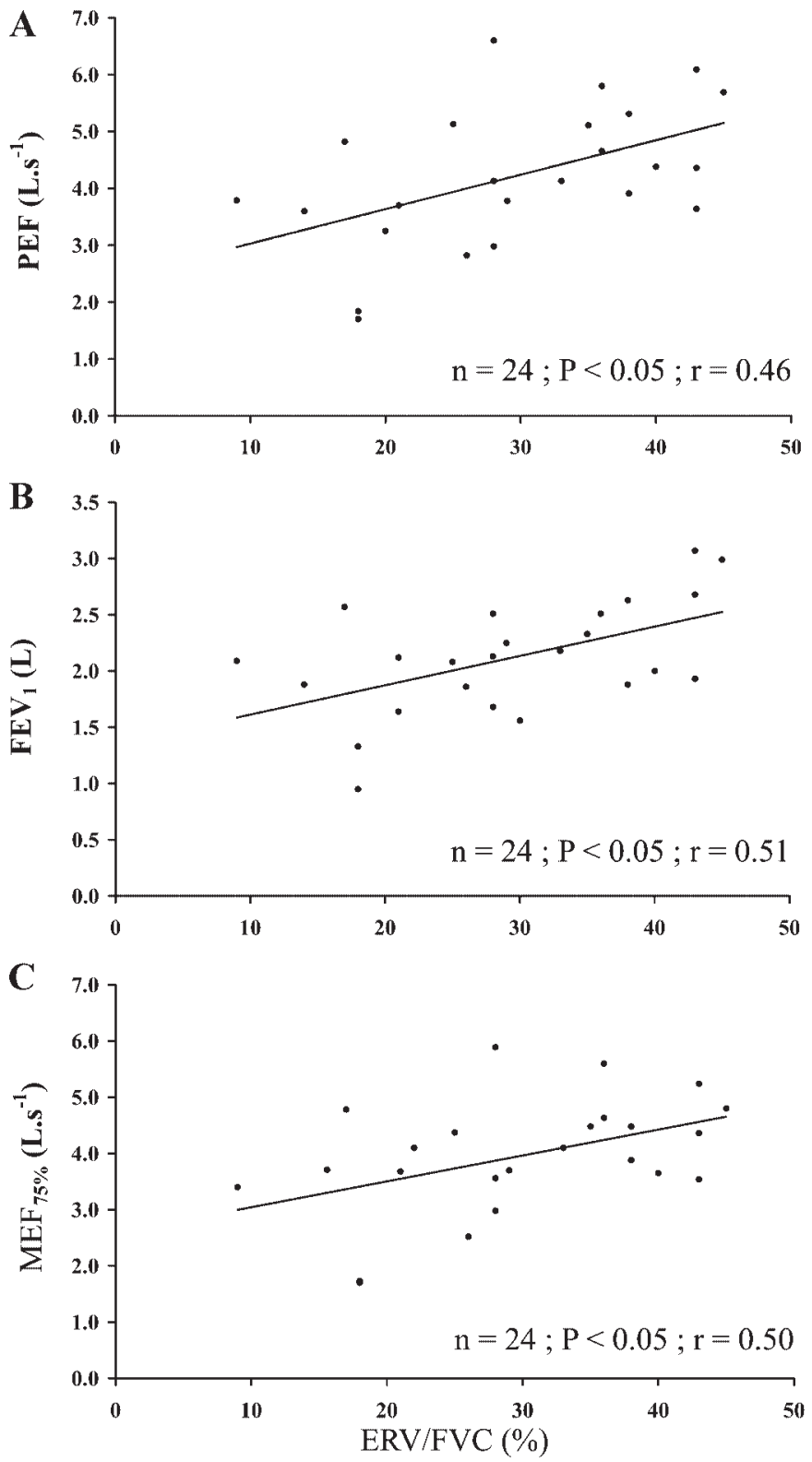

Fig. 6. Significant relationships between peak exercise values of ERV/FVC and $\mathrm{PEF}(A)$, forced expiratory volume in $1 \mathrm{~s}\left(\mathrm{FEV}_{1} ; B\right)$, and $\mathrm{MEF}_{75 \%}(C)$.

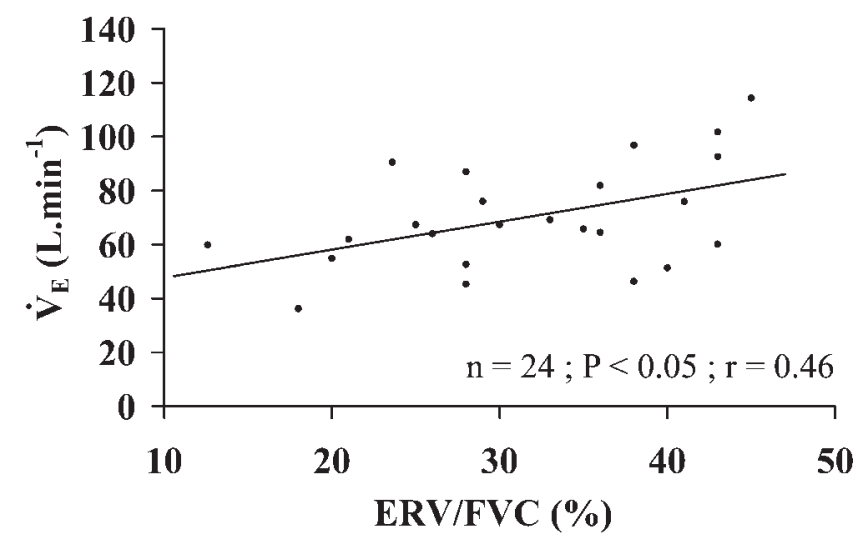

Fig. 7. Significant relationship between peak exercise values of ERV/FVC and minute ventilation ( $\dot{\mathrm{V} E})$. 
tidal breath got closer or met the limit imposed by the MFLV measured at rest. Previous studies in trained adult men, women, and seniors have yet evidenced similar breathing pattern, which suggests that there is an active expiration during which energy is stored in inspiratory muscles $(19,21-23,27)$. At the beginning of the inspiration phase, the release of the energy stored allows the ignition of the inspiration flow to be a passive process (19). Subsequently, as $\dot{V}_{E}$ increased with exercise, Tr increased their EELV up to values near those of rest, which is called dynamical hyperinflation (23). This limits the occurrence of expFL by taking away the exercise tidal breath from the maximal F-V envelope (22). In the Tr group, one child (subject 7) regulated his VT with exercise by both decreasing EELV and increasing EILV up to Ppeak. This breathing pattern is commonly observed in untrained healthy adults and allows dividing the increase in ventilatory work between inspiratory and expiratory muscles $(19,23)$. However, the lack of increase in EELV leaded to an expFL at Ppeak in this child.

In UT, our results show a great variability in the individual regulation process of $\mathrm{V}_{\mathrm{T}}$ during exercise. In this group, six children (subjects 16, 18, 19, 22, 23, and 24) presented nearly the same breathing strategy than trained children, i.e., a decrease followed by an increase in EELV. However, in the five remaining untrained children, the regulation of VT within FVC was more chaotic. In these last children (subjects 14, 15, 17, 20 , and 21), the Vт shifted between the high and the low lung volume without evident logic. For instance, two children presented an expFL without increase in EELV (subjects 17 and 20). This could be explained by thoracic gas compression during the MFVL maneuver or bronchodilatation during exercise. Thoracic gas compression cannot be excluded here and could be responsible for a small part of the expFL in these children. Contrary to trained subjects, it is very difficult to explain why we found heterogeneous breathing patterns in untrained children. One hypothesis could be that their ventilatory demand was not sufficiently high to impose the same breathing pattern in all the untrained subjects. Physical intrasubject difference could be implicated here.

In our study, the differences are not seen between the two groups when comparing them at the same absolute exercise intensity ( 50 and $75 \mathrm{~W}$ ). Nevertheless, this may easily be explained by the fact that the exercise difficulty was not the same between children of the same group for a given exercise intensity. For instance, at $75 \mathrm{~W}$, some children have already passed their respiratory compensation point, whereas other children are still very much under it. In these conditions normalization in percent of the Ppeak seems more representative of the exercise difficulty.

Thus our results suggest that ventilation level is a great determinant of the breathing pattern in children. Indeed, when ventilation demand becomes important, children have to adopt a breathing pattern characterized by an increase in EELV at moderate or strenuous exercise. This seems to be supported by the fact that the children with the highest $\dot{V}_{E}$ are also those who breathe at the highest lung volume (Fig. 7). Maximal expiratory flows seem to be other determinants of the breathing pattern during heavy exercise in children because peak exercise values of ERV/FVC were significantly related to $\mathrm{FEV}_{1}$, PEF, and $\mathrm{MEF}_{75 \%}$ in all subjects. This suggests that trained children, who are also those who presented the higher maximal expira- tory flows, seem more able to take advantage of the higher available maximal expiratory airflow than untrained, to avoid ventilatory limitation imposed by higher ventilation. Nevertheless, the increase in EELV is not free from an energetic standpoint (23). The decrease in inspiratory muscle length due to the increase of EELV at heavy exercise is accompanied by a rise in work and oxygen cost of breathing $(5,21-23)$. By shifting Vт toward the high lung volume, trained and some untrained children keep the tidal breath on the top of the pressure-volume relationship of the lung and chest wall (i.e., the lung compliance is decrease). This results in a higher elastic load of breathing in trained children needing a higher inspiratory drive (20-23). This could explain the higher peak exercise $\mathrm{V}_{\mathrm{T}} / \mathrm{T}_{\mathrm{I}}$ in trained and the relationship between the peak exercise values of this parameter and IRV/FVC in all subjects.

Dyspnea and $S a_{O_{2}}$. In this study, we reported that the $\mathrm{Tr}$ group showed a significant decrease in $\mathrm{Sa}_{\mathrm{O}_{2}}$ at maximal exercise, which was $>4 \%$ in six subjects. In these children, the decrease in $\mathrm{Sa}_{2}$ may likely be explained not only by the normal decreasing $\mathrm{pH}$, increasing temperature, and resultant rightward shift of the $\mathrm{Hb}-\mathrm{O}_{2}$ dissociation curve at high exercise intensity. Thus the higher exercise ventilatory constraints in $\mathrm{Tr}$ could partly explain the decrease in $\mathrm{Sa}_{2}$ in this group and the lower values at Ppeak in Tr than UT. The higher dyspnea in $\mathrm{Tr}$ than UT at Ppeak may be explained by the fact that Tr breathed at higher lung volume. In adults, it has been shown that breathing at high lung volume induces an increase in elastic load, which may explain in this way the higher dyspnea in $\operatorname{Tr}$ $(24,36)$.

In conclusion, even if the occurrence of expiratory flow limitation seems to be nearly the same in trained and untrained subjects, this study supports the hypothesis of higher ventilatory constraints in trained children. Indeed, trained children presented lower BR and breathed at higher lung volumes than untrained at peak exercise. This seems to be imposed by the higher ventilatory demand in Tr than UT and may negatively influence the $\mathrm{Sa}_{\mathrm{O}_{2}}$ level and dyspnea during strenuous exercise.

\section{ACKNOWLEDGMENTS}

We thank the Beuvry hospital, which welcomed us for the experimentations, and all the children from the Jean Monnet primary Illies School who participated enthusiastically in this study. We also thank the Inspection d'Académie of Lille (France).

\section{REFERENCES}

1. American Thoracic Society. Standardization of spirometry-1987 update. Official statement of American Thoracic Society. Respir Care 32: 1039-1060, 1987.

2. Andersen KL, Seliger V, Rutenfranz J, and Messel S. Physical performance capacity of children in Norway. III. Respiratory responses to graded exercise loadings-population parameters in a rural community. Eur J Appl Physiol 33: 265-274, 1974.

3. Andrew GM, Becklake MR, Guleria JS, and Bates DV. Heart and lung functions in swimmers and nonathletes during growth. J Appl Physiol 32: 245-251, 1972.

4. Babb TG. Breathing $\mathrm{He}-\mathrm{O}_{2}$ increases ventilation but does not decrease the work of breathing during exercise. Am J Respir Crit Care Med 163: 1128-1134, 2001.

5. Babb TG. Mechanical ventilatory constraints in aging, lung disease, and obesity: perspectives and brief review. Med Sci Sports Exerc 31: S12-S22, 1999

6. Babb TG, Buskirk ER, and Hodgson JL. Exercise end-expiratory lung volumes in lean and moderately obese women. Int J Obes 13: 11-19, 1989. 
7. Borg GA. Psychophysical bases of perceived exertion. Med Sci Sports Exerc 14: 377-381, 1982.

8. Cooper DM, Kaplan MR, Baumgarten L, Weiler-Ravell D, Whipp BJ, and Wasserman $\mathbf{K}$. Coupling of ventilation and $\mathrm{CO}_{2}$ production during exercise in children. Pediatr Res 21: 568-572, 1987.

9. Courteix D, Obert P, Lecoq AM, Guenon P, and Koch G. Effect of intensive swimming training on lung volumes, airway resistance and on the maximal expiratory flow-volume relationship in prepubertal girls. Eur J Appl Physiol 76: 264-269, 1997.

10. Deheeger M, Rolland-Cachera MF, and Fontvieille AM. Physical activity and body composition in 10 year old French children: linkages with nutritional intake? Int J Obes Relat Metab Disord 21: 372-379, 1997.

11. Dempsey JA and Johnson BD. Demand vs. capacity in the healthy pulmonary system. Schweiz Z Sportmed 40: 55-64, 1992.

12. Dempsey JA and Wagner PD. Exercise-induced arterial hypoxemia. J Appl Physiol 87: 1997-2006, 1999.

13. Durnin JV and Rahaman MM. The assessment of the amount of fat in the human body from measurements of skinfold thickness. Br J Nutr 21 681-689, 1967.

14. Ekblom B. Effect of physical training in adolescent boys. J Appl Physio 27: $350-355,1969$.

15. Gratas-Delamarche A, Mercier J, Ramonatxo M, Dassonville J, and Prefaut C. Ventilatory response of prepubertal boys and adults to carbon dioxide at rest and during exercise. Eur J Appl Physiol 66: 25-30, 1993.

16. Hamilton $\mathbf{P}$ and Andrew GM. Influence of growth and athletic training on heart and lung functions. Eur J Appl Physiol 36: 27-38, 1976.

17. Harms CA, McClaran SR, Nickele GA, Pegelow DF, Nelson WB, and Dempsey JA. Effect of exercise-induced arterial $\mathrm{O}_{2}$ desaturation on $\dot{\mathrm{VO}}_{2 \max }$ in women. Med Sci Sports Exerc 32: 1101-1108, 2000.

18. Harms CA, McClaran SR, Nickele GA, Pegelow DF, Nelson WB, and Dempsey JA. Exercise-induced arterial hypoxaemia in healthy young women. J Physiol 507: 619-628, 1998.

19. Henke KG, Sharratt M, Pegelow D, and Dempsey JA. Regulation of end-expiratory lung volume during exercise. J Appl Physiol 64: 135-146, 1988.

20. Johnson BD, Reddan WG, Pegelow DF, Seow KC, and Dempsey JA Flow limitation and regulation of functional residual capacity during exercise in a physically active aging population. Am Rev Respir Dis 143: 960-967, 1991.

21. Johnson BD, Reddan WG, Seow KC, and Dempsey JA. Mechanical constraints on exercise hyperpnea in a fit aging population. Am Rev Respir Dis 143: 968-977, 1991.

22. Johnson BD, Saupe KW, and Dempsey JA. Mechanical constraints on exercise hyperpnea in endurance athletes. J Appl Physiol 73: 874-886, 1992.
23. Johnson BD, Weisman IM, Zeballos RJ, and Beck KC. Emerging concepts in the evaluation of ventilatory limitation during exercise: the exercise tidal flow-volume loop. Chest 116: 488-503, 1999.

24. Jones NL. Dyspnea in exercise. Med Sci Sports Exerc 16: 14-19, 1984.

25. Knudson RJ, Lebowitz MD, Holberg CJ, and Burrows B. Changes in the normal maximal expiratory flow-volume curve with growth and aging. Am Rev Respir Dis 127: 725-734, 1983.

26. Lussier $\mathbf{L}$ and Buskirk ER. Effects of an endurance training regimen on assessment of work capacity in prepubertal children. Ann NY Acad Sci 301: 734-747, 1977.

27. McClaran SR, Harms CA, Pegelow DF, and Dempsey JA. Smaller lungs in women affect exercise hyperpnea. J Appl Physiol 84: 1872-1881, 1998.

28. McClaran SR, Wetter TJ, Pegelow DF, and Dempsey JA. Role of expiratory flow limitation in determining lung volumes and ventilation during exercise. J Appl Physiol 86: 1357-1366, 1999.

29. Mead J. Dysanapsis in normal lungs assessed by the relationship between maximal flow, static recoil, and vital capacity. Am Rev Respir Dis 121: $339-342,1980$.

30. Nourry C, Fabre C, Bart F, Grosbois JM, Berthoin S, and Mucci P. Evidence of exercise-induced arterial hypoxemia in prepubescent trained children. Pediatr Res 55: 674-681, 2004.

31. Prioux J, Matecki S, Amsallem F, Denjean A, and Ramonatxo $M$. [Ventilatory response to maximal exercise in the normal child]. Rev Mal Respir 20: 904-911, 2003.

32. Rowland TW and Cunningham LN. Development of ventilatory responses to exercise in normal white children. A longitudinal study. Chest 111: 327-332, 1997

33. Stein R, Selvadurai H, Coates A, Wilkes DL, Schneiderman-Walker J, and Corey M. Determination of maximal voluntary ventilation in children with cystic fibrosis. Pediatr Pulmonol 35: 467-471, 2003.

34. Sundberg $\mathbf{S}$ and Elovainio R. Cardiorespiratory function in competitive endurance runners aged 12-16 years compared with ordinary boys. Acta Paediatr Scand 71: 987-992, 1982.

35. Tanner J. Growth at Adolescence. Oxford, UK: Blackwell Scientific, 1962, p. 325.

36. Younes M. Load responses, dyspnea, and respiratory failure. Chest 97 : 59S-68S, 1990.

37. Zinman R and Gaultier C. Maximal static pressures and lung volumes in young female swimmers. Respir Physiol 64: 229-239, 1986.

38. Zinman R and Gaultier C. Maximal static pressures and lung volumes in young female swimmers: one year follow-up. Pediatr Pulmonol 3: 145$148,1987$. 